\title{
CORRELAÇÃO ENTRE O ENVELHECIMENTO E AS DIMENSÕES DAS PREGAS VOCAIS
}

\section{Correlation between aging and vocal folds' dimensions}

\author{
Sandrelli Virginio de Vasconcelos Miranda ${ }^{(1)}$, Roberto José Vieira de Mello ${ }^{(2)}$, \\ Hilton Justino da Silva ${ }^{(3)}$
}

\begin{abstract}
RESUMO
Objetivo: avaliar as dimensões das pregas vocais em ambos os sexos e correlacioná-las com o envelhecimento. Método: foram estudados 30 cadáveres (15 mulheres e 15 homens) na faixa etária de 60 a 102 anos. Para isto foram seguidas quatro etapas sequenciais: $1^{a}$ ) História clínica do cadáver; $2^{a}$ ) Remoção da laringe; $3^{a}$ ) Dissecação da laringe; 4ㄹ) Morfometria das dimensões das pregas vocais. Trata-se de um estudo experimental. Resultados: o comprimento da prega vocal masculina com média de 15,90 mm foi maior que o da feminina com 10,39 mm. A largura da prega vocal masculina foi maior com média de 2,37 mm que a feminina com 2,31 mm. A média geral da espessura também foi maior no sexo masculino com média de $2,55 \mathrm{~mm}$ em comparação com a feminina com 2,38 $\mathrm{mm}$. Não foram encontradas diferenças estatísticas entre a idade e o comprimento, largura e espessura das pregas vocais em ambos os sexos. Conclusão: as dimensões de comprimento, largura e espessura das pregas vocais foram maiores no sexo masculino. Entretanto, não houve diferença, estatisticamente significante, entre as três dimensões das pregas vocais durante o envelhecimento em ambos os sexos.
\end{abstract}

DESCRITORES: Envelhecimento; Idoso; Cordas Vocais; Voz; Anatomia

\section{INTRODUÇÃO}

O envelhecimento é um processo dinâmico e progressivo, no qual há modificações em diversos aspectos. Este processo é determinado por vários fatores que estão presentes desde o nascimento e vão se desenvolvendo ao longo da vida, e tem uma grande variação intraindividual e interindividual ${ }^{1}$.

As estruturas laríngeas envelhecem concomitantemente a todos os tecidos do corpo a partir dos 60 anos de idade ${ }^{2}$ diferindo em muitos aspectos característicos da laringe do adulto jovem ${ }^{3}$.

(1) Fonoaudióloga da Prefeitura Municipal de Maceió, AL, Brasil; e da Secretaria Estadual de Educação de Pernambuco, Recife, PE, Brasil; Mestre em Patologia (Morfologia Aplicada) pela Universidade Federal de Pernambuco.

(2) Patologista; Professor Associado I da Universidade Federal de Pernambuco, UFPE, Recife, PE, Brasil; Doutor em Medicina pela Universidade Federal de Pernambuco.

(3) Fonoaudiólogo; Professor Adjunto I da Universidade Federal de Pernambuco, UFPE, Recife, PE, Brasil; Doutor em Nutrição pela Universidade Federal de Pernambuco.

Conflito de interesses: inexistente
As alterações anatômicas e fisiológicas do trato vocal são frequentemente subestimadas ou não reconhecidas e o conhecimento destas alterações na laringe durante o processo de envelhecimento é crucial $^{4}$. Pois, a presbifonia é uma das causas mais comuns de rouquidão e distúrbios de voz na população idosa ${ }^{5}$.

Dentre outros fatores anatômicos e fisiológicos que estão envolvidos no envelhecimento laríngeo, dois tipos de alterações anatômicas são mais destacados: a calcificação e ossificação gradual das cartilagens laríngeas, que causam diminuição da mobilidade laríngea e redução na excursão das cartilagens aritenóides.

Num estudo 6 com imagens laringoscópicas, foi observado que a maior parte da musculatura das pregas vocais diminui caracterizada pela atrofia dos músculos intrínsecos da laringe, redução da espessura da prega vocal e alterações na qualidade de contração muscular.

A atrofia da prega vocal do idoso é uma parte do processo de envelhecimento normal, presente em até $60 \%$ de indivíduos após os 60 anos 
apresentando evidências de insuficiência glótica ${ }^{7}$. A literatura também refere redução da vibração das pregas vocais devido à diminuição da saliva e de secreções mucosas na laringe do idoso $0^{6}$. Além disso, as alterações histológicas dos fibroblastos das pregas vocais são uma das principais causas da mudança na qualidade da voz com o envelhecimento ${ }^{8}$.

Estas alterações morfológicas causadas pelo envelhecimento causam transtornos vocais como: tempo máximo de fonação diminuído; redução da velocidade de fala; diminuição da extensão vocal e dificuldade no canto? ${ }^{2}$.

Além da presbifonia, a rouquidão nos idosos pode ser causada por: lesões benignas da prega vocal $(28,6 \%)$, seguido de lesões malignas $(27,6 \%)$, paralisias de pregas vocais $(25,5 \%)$, refluxo laringofaríngeo $(13,3 \%)$ e disfonia funcional $(10,2 \%)^{4}$.

Alguns estudos morfométricos foram realizados com a laringe ${ }^{9,10}$ e com as pregas vocais ${ }^{11,12}$. No entanto, poucos estudos investigaram as dimensões de comprimento, largura e espessura e as correlacionaram com o envelhecimento em ambos os sexos. Sendo assim, mostra-se relevante ampliar os estudos neste sentido.

Desta maneira, o objetivo geral desta pesquisa foi avaliar as dimensões das pregas vocais em ambos os sexos e correlacioná-las com o envelhecimento.

\section{MÉTODO}

Fizeram parte deste estudo 30 cadáveres, sendo 15 do sexo masculino e 15 do sexo feminino na faixa etária de 60 a 102 anos, provenientes do SVO - Serviço de Verificação de Óbitos. Tratou-se de um estudo experimental.

Os casos selecionados obedeceram a critérios de inclusão e exclusão. Foram incluídos na pesquisa indivíduos vítimas de morte não-violenta, com até 24 horas post-mortem e idade a cima dos 60 anos.

Foram excluídos os indivíduos abaixo dos 60 anos submetidos previamente à traqueostomia, cirurgia laríngea, radioterapia na região cérvicofacial, acometidos por neoplasias benignas ou malignas na região tóraco-cervical ou qualquer patologia que afetasse a região laríngea.

Para a coleta de dados foram seguidas quatro etapas sequenciais:

1a) História clínica do cadáver: foram feitas anotações no protocolo de morfometria das pregas vocais sobre data do óbito, hora, idade, data de nascimento, sexo, história clínica, hábitos (fumo e álcool) e profissão.

$2^{a}$ ) emoção da laringe: após seleção e preenchimento do protocolo, foi realizada durante a necropsia do cadáver, a remoção da laringe através de incisão na pele da região torácica alta e levantamento do retalho cutâneo tóracocervical. A laringe foi removida em bloco após secção sobre o osso hióide e ao nível do quarto anel traqueal.

$3^{\text {a) }}$ Dissecação da laringe: a laringe foi dissecada cuidadosamente sendo removidos a musculatura extrínseca da laringe, vasos e nervos.

$4^{a}$ ) Morfometria das dimensões das pregas vocais: as laringes dissecadas não foram fixadas por nenhum tipo de substância, de forma a evitar deformações no tecido e alterações nas medidas.

Os pontos de localização entre as dimensões da porção membranosa das pregas vocais foram baseados em outro estudo morfométrico ${ }^{11} \mathrm{e}$ as medidas realizadas pela mesma pesquisadora de forma a evitar vícios.

As medidas foram realizadas em milímetros com paquímetro digital Stainless Hardened de acurácia $0,01 \mathrm{~mm}$ e foram anotadas no protocolo de morfometria das pregas vocais (Figura 3 ).

A ordem das medidas foi feita na seguinte sequência: largura, espessura e comprimento em ambos os lados direito e esquerdo respectivamente.

O comprimento foi medido com a haste de profundidade do paquímetro digital com localização desde a inserção da prega vocal na cartilagem tireóide, inferiormente ao pecíolo da epiglote até a sua inserção no processo vocal da cartilagem aritenóide.

A largura foi medida com a haste externa do paquímetro digital com localização definida como a distância compreendida entre a borda livre e a transição entre o ligamento vocal e o assoalho do ventrículo laríngeo.

A espessura foi medida com a haste externa do paquímetro digital com localização definida como a distância entre dois planos paralelos traçados superior e inferiormente ao músculo tireoaritenóide (TA).

Os dados coletados foram organizados no Microsoft Excel 2003 e distribuídos em tabelas e gráficos para melhor visualização. Para a análise dos dados foi empregada estatística descritiva para diferenciação das dimensões em ambos os sexos e foi pesquisada a existência de correlação entre a idade e as respectivas dimensões das pregas vocais utilizando o Coeficiente de Correlação de Pearson e de Spearman. Foi considerado índice de significância de $95 \%(p<0,05)$. O programa estatístico utilizado foi o BioStat versão 5.0.

Por considerações éticas, esta pesquisa foi aprovada pelo Comitê de Ética em Seres Humanos com registro № 309/07. 


\section{RESULTADOS}

A média da idade de idosos do sexo feminino foi de 74,46 anos, variando de 61 a 102 anos. Já no sexo masculino a média foi de 71,52 anos com idades entre 61 a 81 anos.

$\mathrm{Na}$ Tabela 1, observam-se os valores da média, valor mínimo, valor máximo e o desvio-padrão das dimensões das pregas vocais no sexo feminino. O comprimento variou de 8,92 a $12,20 \mathrm{~mm}$, com média de 10,36 mm na prega vocal direita, e 8,65 a $11,94 \mathrm{~mm}$, na prega vocal esquerda com média de 10,43 mm. A largura variou de 1,91 a 3,01 na prega vocal direita com média de $2,47 \mathrm{~mm}$ e 1,55 a 2,66mm na prega vocal esquerda com média de $2,16 \mathrm{~mm}$. A espessura variou entre 1,38 a 3,43 na prega vocal direita com média de $2,46 \mathrm{~mm}$ e 1,41 a 3,08 na prega vocal esquerda com média de 2,30 mm.

Na Figura 1, observa-se por meio do teste de correlação de Pearson, a correlação da idade e as três dimensões das pregas vocais no sexo feminino. Não houve diferença estatística significante entre o aumento da idade e as três dimensões das pregas vocais: comprimento $(p=0,41)$, largura $(p=0,47)$ e espessura $(p=0,13)$.

Tabela 1 - Dimensões das pregas vocais em idoso do sexo feminino

\begin{tabular}{lcccc}
\hline & Valor Média & Valor Mínimo & Valor Máximo & $\begin{array}{c}\text { Desvio-Padrão } \\
\text { (DV) }\end{array}$ \\
\hline Cpvd & 10,36333 & 8,92 & 12,20 & 1,027588 \\
Cpve & 10,43333 & 8,65 & 11,94 & 1,080051 \\
Lpvd & 2,474667 & 1,91 & 3,01 & 0,350405 \\
Lpve & 2,158 & 1,55 & 2,66 & 0,336138 \\
Epvd & 2,465333 & 1,38 & 3,43 & 0,485943 \\
Epve & 2,304 & 1,41 & 3,08 & 0,459702 \\
\hline
\end{tabular}

Legenda: Cpvd= Comprimento da prega vocal direita; Cpve= Comprimento da prega vocal esquerda; Lpvd= Largura da prega vocal direita; Lpve= Largura da prega vocal esquerda; Epvd= Espessura da prega vocal direita; Epve= Espessura da prega vocal esquerda.

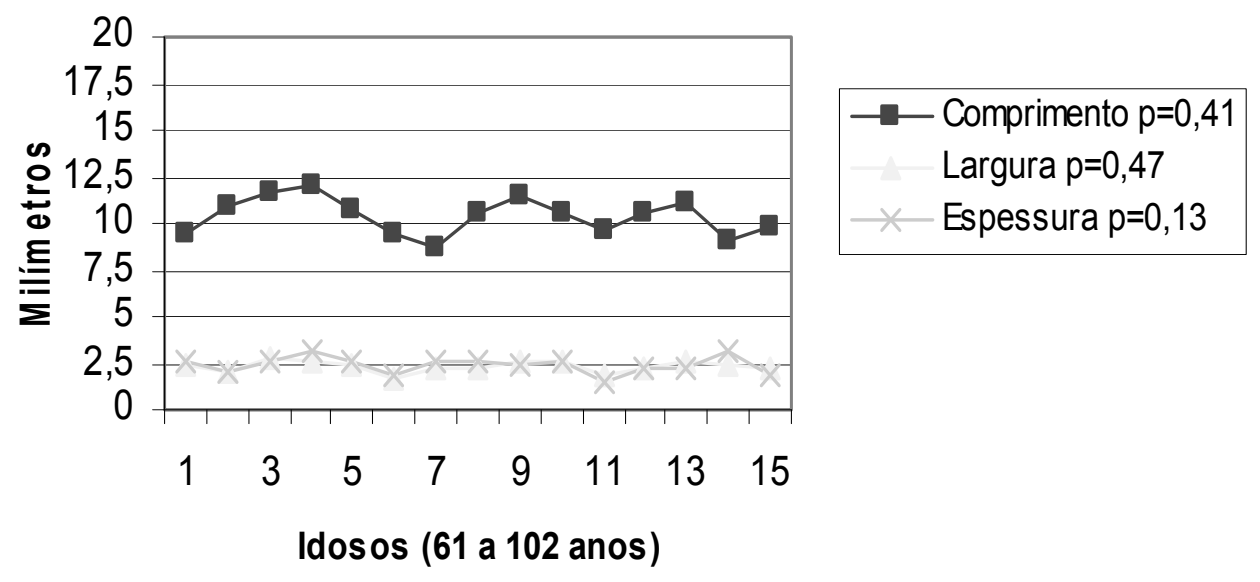

Figura 1 - Correlação entre o envelhecimento e as dimensões das pregas vocais em idosos do sexo feminino

$\mathrm{Na}$ Tabela 2, pode-se observar os valores da média, valor mínimo, valor máximo e o desviopadrão das dimensões das pregas vocais no sexo masculino. O comprimento variou de 12,31 a 18,35 $\mathrm{mm}$, na prega vocal direita com média de 15,46 $\mathrm{mm}$, e 12,91 a 19,49 mm com média de 16,34 mm na prega vocal esquerda. O comprimento foi maior na prega vocal esquerda. A largura variou de 1,54 a $3,22 \mathrm{~mm}$ com média de $2,52 \mathrm{~mm}$ na prega vocal direita e 0,73 a 2,73 $\mathrm{mm}$ na prega vocal esquerda com média de 2,64 mm. Desta maneira, a prega vocal esquerda apresentou largura maior que a 
direita. A espessura variou entre 1,61 a 3,33 mm na prega vocal direita com média de $2,46 \mathrm{~mm}$, e 1,46 a $3,70 \mathrm{~mm}$, na prega vocal esquerda com média de 2,64 $\mathrm{mm}$. Logo, houve uma assimetria entre a espessura das pregas vocais, maior do lado esquerdo.

$\mathrm{Na}$ Figura 2 visualiza-se a correlação das dimensões das pregas vocais com a idade no sexo masculino. Não houve diferença significante no comprimento $(p=0,68)$ e na espessura $(p=0,93)$ realizado pelo teste de correlação de Pearson. A análise da correlação da idade com a largura foi feita pelo coeficiente de Spearman e também não houve significância entre os achados $(p=0,14)$.

Tabela 2 - Dimensões das pregas vocais em idosos do sexo masculino

\begin{tabular}{lcccc}
\hline & Valor Média & Valor Mínimo & Valor Máximo & $\begin{array}{c}\text { Desvio-Padrão } \\
\text { (DV) }\end{array}$ \\
\hline Cpvd & 15,464 & 12,31 & 18,35 & 2,000753 \\
Cpve & 16,34067 & 12,91 & 19,49 & 2,053118 \\
Lpvd & 2,52 & 1,54 & 3,22 & 0,507979 \\
Lpve & 2,217333 & 0,73 & 2,73 & 0,528846 \\
Epvd & 2,463333 & 1,61 & 3,33 & 0,481763 \\
Epve & 2,636 & 1,46 & 3,70 & 0,565948 \\
\hline
\end{tabular}

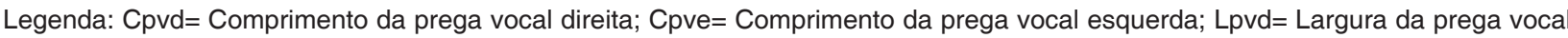
direita; Lpve= Largura da prega vocal esquerda; Epvd= Espessura da prega vocal direita; Epve= Espessura da prega vocal esquerda.

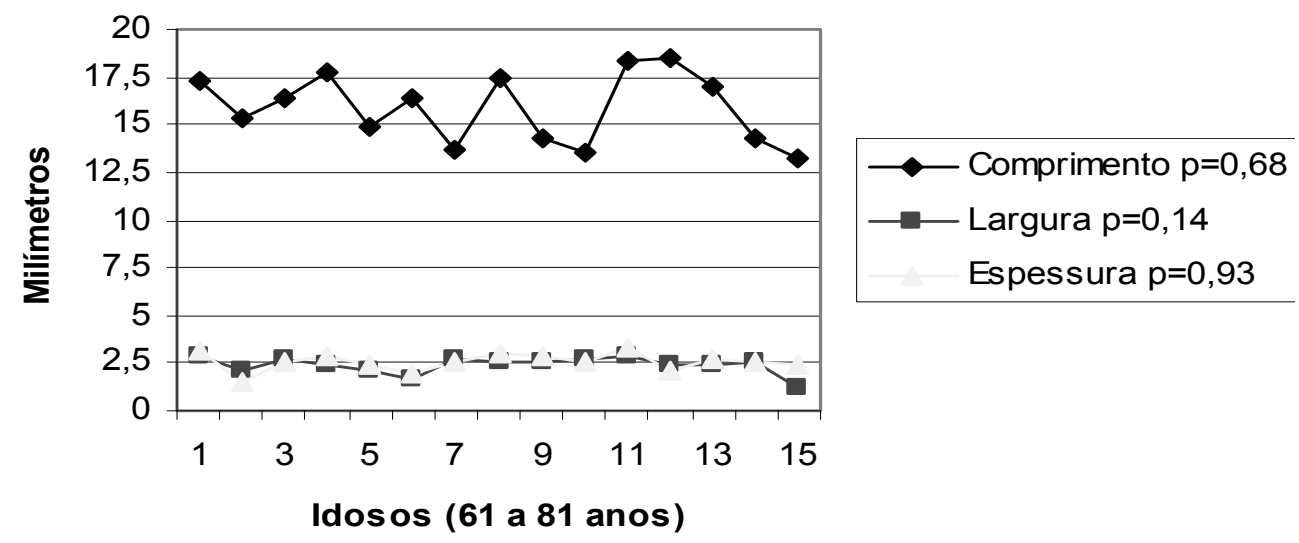

Figura 2 - Correlação entre o envelhecimento e as dimensões das pregas vocais em idosos do sexo masculino

$\mathrm{Na}$ Tabela 3, observam-se os valores da média das três dimensões das pregas vocais nos idosos em ambos os sexos. O comprimento da prega vocal masculina com média de $15,90 \mathrm{~mm}$ foi maior que o da feminina com 10,39 mm. A largura das pregas vocais masculinas apresentou média maior com $2,37 \mathrm{~mm}$ que as femininas com 2,31 $\mathrm{mm}$. A média geral da espessura também foi maior no sexo masculino com média de $2,55 \mathrm{~mm}$ em comparação com a feminina com $2,38 \mathrm{~mm}$.
Tabela 3 - Média das dimensões das pregas vocais em idosos do sexo feminino e masculino

\begin{tabular}{ccc}
\hline $\begin{array}{c}\text { Média } \\
\text { Dimensão }\end{array}$ & Feminino & Masculino \\
\hline MCpv & 10,395 & 15,90233 \\
MLpv & 2,31 & 2,368667 \\
MEpv & 2,38 & 2,549667 \\
\hline
\end{tabular}

Legenda: $\mathrm{MCpv}=$ Média do Comprimento das pregas vocais; $\mathrm{MLpv}=$ Média da Largura das pregas vocais; MEpv= Média da Espessura das pregas vocais. 


\section{DISCUSSÃO}

Em geral, as maiores mudanças na laringe de idosos se iniciam aos 60 anos no homem e após a menopausa na mulher ${ }^{6}$.

Quanto às três dimensões das pregas vocais, na Tabela 1 foi observado que, no sexo feminino, o valor médio do comprimento da prega vocal direita foi de $10,36 \mathrm{~mm}$ e na esquerda de $10,43 \mathrm{~mm}$, sendo o comprimento entre as pregas direita e esquerda maior do lado esquerdo. A Tabela 2 aponta que nos homens o comprimento da prega vocal direita foi de $15,46 \mathrm{~mm}$ e na esquerda de $16,34 \mathrm{~mm}$, havendo também uma diferença maior do lado esquerdo, sugerindo uma possível assimetria entre a prega vocal direita e esquerda em ambos os sexos.

$\mathrm{Na}$ Tabela 3, os valores médios do comprimento entre os sexos, mostraram uma diferença entre o comprimento das pregas vocais de homens $(15,90$ $\mathrm{mm}$ ) e mulheres $(10,39 \mathrm{~mm})$ já mencionados na literatura. Esta diferença morfológica entre o comprimento das pregas vocais afeta a freqüência da voz. Um estudo sobre análise acústica da voz do idoso verificou que há uma diferença significante entre as medidas da freqüência fundamental de idosos do sexo masculino e feminino. Nas mulheres há um declínio mais acentuado, que não interfere no julgamento do sexo ${ }^{13}$.

Um trabalho ${ }^{13}$ abordou a correlação entre a altura corporal e as dimensões das pregas vocais sendo estas mensuradas com paquímetro em 24 cadáveres de ambos os sexos na faixa etária de 29 a 90 anos e se encontrou pregas vocais masculinas maiores que as femininas. A maioria dos estudos encontrados foram comparativos entre adultos e idosos, portanto, não investigaram uma população homogênea de idosos.

Não foram encontradas referências que reforcem a assimetria entre as pregas vocais dos idosos e sua etiologia. Entretanto, acredita-se que as estas alterações citadas anteriormente poderiam causar esta assimetria entre o comprimento das pregas vocais, o que nos achados foi mais evidente entre os homens. Outros ${ }^{12,14}$ relatam que as características glóticas presentes como as citadas anteriormente além de terem uma forte correlação entre si, sua ocorrência é semelhante entre homens e mulheres.

Em relação à largura, a média da prega vocal direita na mulher foi de $2,47 \mathrm{~mm}$ e na esquerda de $2,15 \mathrm{~mm}$, no homem a largura da prega vocal direita teve uma média de 2,52 $\mathrm{mm}$ e na esquerda de 2,21 $\mathrm{mm}$. Em ambos os lados, encontra-se diferença entre homens e mulheres, sendo maior a largura das pregas vocais nos homens e do lado direito em ambos os sexos. Numa publicação com morfometria por paquímetro ${ }^{11} \mathrm{com}$ adultos e idosos de ambos os sexos também foi observada a largura das pregas vocais dos homens maior que nas mulheres.

Poucos estudos abordando diretamente a dimensão de largura das pregas vocais na população idosa foram encontrados. Autores destacam que devido a questões hormonais, durante a perimenopausa, a mulher já não é mais capaz de manter a tonicidade e resistência dos músculos da prega vocal. E uma conseqüência disso é a atrofia progressiva das duas pregas vocais. A membrana mucosa que as cobre se torna fina e desidratada ${ }^{6}$.

A espessura das pregas vocais no sexo feminino foi de 2,46 $\mathrm{mm}$ na prega vocal direita, e 2,30 $\mathrm{mm}$ na esquerda. No sexo masculino também foi de 2,46 $\mathrm{mm}$ na direita e $2,63 \mathrm{~mm}$ na esquerda. Em ambos os sexos ocorreram assimetrias, mas não houve correspondência entre os lados direito e esquerdo. Segundo demonstrado na Tabela 3, a média da espessura foi maior nos homens $(2,54 \mathrm{~mm})$ que nas mulheres $(2,38 \mathrm{~mm})$.

Na Figura 1 há uma representação da correlação da idade com as dimensões das pregas vocais no sexo feminino e na figura 2 no sexo masculino. Não houve mudanças estatisticamente significantes nas três dimensões durante o envelhecimento em ambos os sexos.

Quanto ao comprimento, num estudo histológico ${ }^{2}$ com 64 laringes de idosos entre 70 e 104 anos, foi observado um encurtamento da prega vocal membranosa no sexo masculino.

Sobre a largura das pregas vocais durante o envelhecimento, foi encontrado um engrossamento da mucosa no sexo feminino ${ }^{2}$. No sexo masculino relataram que a camada intermediária da lâmina própria teve seu contorno deteriorado, as fibras elásticas se tornaram menos densas e atrofiaram, a camada profunda da lâmina própria engrossou, as fibras colágenas ficaram densas e fibróticas. Em ambos os sexos, declararam presença de edema na camada superficial da lâmina própria.

Outros autores ${ }^{12}$ apontaram uma redução da espessura da lâmina própria $(p<0.001)$ e da densidade de células epiteliais ( $p<0.001)$, em função da idade e essas mudanças foram mais freqüentes nos homens. A atrofia é responsável por graves perturbações na qualidade vocal ${ }^{15}$ e o número de pacientes idosos com atrofia de prega vocal aumenta gradualmente quase todos os anos, sendo que os pacientes do sexo masculino são responsáveis por $65 \%$ dessa população ${ }^{16}$.

A virilização da voz nas mulheres idosas é causada pela diminuição da atividade ovariana que leva a uma redução drástica dos níveis de progesterona e estrógeno. Além disso, concomitantemente, há uma queda considerável na secreção de 


\section{PROTOCOLO DE MORFOMETRIA DAS PREGAS VOCAIS}

№

DATA:

HORA:

IDADE:
SEXO: $\mathrm{M}(\quad) \mathrm{F}(\mathrm{I})$

HISTÓRIA CLÍNICA:

\section{HÁBITOS}

FUMO: SIM ( ) NÃO ( )

ÁLCOOL: SIM ( ) NÃO ( )

PROFISSÃO:

Obs:

\section{MORFOMETRIA}

\section{PREGA VOCAL DIREITA}

COMPRIMENTO (HP):

LARGURA (HE):

ESPESSURA (HE):

\section{PREGA VOCAL ESQUERDA}

COMPRIMENTO (HP):

LARGURA (HE):

ESPESSURA (HE):

\section{Legenda:}

$\mathrm{HP}=$ Haste de Profundidade do paquímetro digital

$\mathrm{HE}=$ Haste Externa do paquímetro digital

Figura 3 - Protocolo de morfometria das pregas vocais

hormônios masculinos como a testosterona e como estes já não são mais contrabalançados pelos hormônios femininos há a redução da freqüência fundamental feminina, deixando a voz mais grave ${ }^{6}$. Havendo também nas mulheres diminuição dos tempos máximos de fonação nas vogais ${ }^{17}$
Pois, declinações nos mecanismos respiratórios e da laringe podem ocorrer simultaneamente no envelhecimento feminino ${ }^{18}$.

Numa revisão bibliográfica ${ }^{19}$ sobre as alterações morfológicas no envelhecimento do músculo TA, considerado o principal músculo da produção da 
voz, os autores referem que a maioria dos estudos encontrou atrofia no músculo, mas há divergências sobre os tipos de fibras perdidas, se tipo I, II ou ambas, sendo necessárias mais investigações sobre o assunto. A utilização da videolaringoestroboscopia permite um diagnóstico preciso da disfonia relacionada à idade ${ }^{3}$. Este exame permite distinguir uma disfonia orgânica de uma atrofia de pregas vocais ${ }^{15}$.

Alguns estudos mostraram que mesmo com a alteração na qualidade vocal pelo envelhecimento há uma auto-imagem vocal positiva por parte dos idosos ${ }^{20}$ e que esta mudança na voz não interfere na sua comunicação quando estão residindo em instituições de longa permanência ${ }^{21}$.

No entanto, no envelhecimento há doenças associadas à disfonia como por exemplo a perda auditiva. Os idosos com perda auditiva são mais propensos a terem disfonia, e com isso desenvolvem um quadro depressivo, causando impacto na qualidade de vida ${ }^{22}$.

Por isso, os fatores fisiológicos envolvidos no envelhecimento dos tecidos da laringe e os possíveis tratamentos clínicos devem ser estudados ${ }^{23}$ e os esforços devem ser direcionados para elucidação da causa ${ }^{4}$.

\section{CONCLUSÃO}

Em relação à correlação entre o envelhecimento e as dimensões das pregas vocais em homens e mulheres se conclui que:

As dimensões de comprimento, largura e espessura das pregas vocais apresentaram tendência a serem maiores em valores absolutos nos idosos do sexo masculino.

A prega vocal apresentou comprimento maior do lado esquerdo e a largura foi maior do lado direito em ambos os sexos. Já a espessura variou de lado sendo maior na prega vocal direita das mulheres e esquerda dos homens. Ocorrendo assim assimetria entre as pregas vocais.

Não houve diferença, estatisticamente significante, entre as três dimensões das pregas vocais com o aumento da idade em ambos os sexos.

\begin{abstract}
Purpose: to evaluate the dimensions of vocal folds in both genders and correlate them with aging. Method: we studied 30 cadavers (15 men and 15 women) from 60 to 102 year-old. For this purpose we followed four steps: 1st) History of the body; 2nd) Removal of the larynx, 3rd) Dissection of the larynx, 4th) Morphometry for the dimensions of the vocal folds. This is an experimental study. Results: the length of the vocal male with an average of $15.90 \mathrm{~mm}$ was higher than the female with $10.39 \mathrm{~mm}$. The width of the male vocal was higher with an average of $2.37 \mathrm{~mm}$ to $2.31 \mathrm{~mm}$ with a female. The overall average thickness was also higher in males with an average of $2.55 \mathrm{~mm}$ compared with women with $2.38 \mathrm{~mm}$. No differences were found among age and the length, width and thickness of the vocal folds in both genders. Conclusion: the dimensions of length, width and thickness of the vocal folds were higher in males. But, there was no statistically significant difference among the three dimensions of the vocal folds during aging in both genders.
\end{abstract}

KEYWORDS: Aging; Aged; Vocal Cords; Voice; Anatomy

\section{REFERÊNCIAS}

1. Brasolotto AG. Voz na terceira idade. In: Ferreira LP, Befi-Lopes DM, Limongi SCO. Tratado de fonoaudiologia. São Paulo: Roca; 2004. p 127-37.

2. Martins RHG. A voz e seus distúrbios. São Paulo: Cultura Acadêmica; 2005.

3. Pontes P, Yamasaki R, Behlau M. Morphological and Functional Aspects of the Senile Larynx. Folia Phoniatr Logop. 2006; 58:151-8.

4. Kandoğan T. Presbylarynges, as a common benign cause of dysphonia in the elderly. Turkish $\mathrm{J}$ Geriatrics. 2003; 6(2):68-71.

5. Roy N, Stemple J, Merrill RM, Thomas L. Epidemiology of voice disorders in the elderly: preliminary findings. Laryngoscope. 2007 Apr;117(4):628-33.

6. Benninger MS, Abitbol J. Voice: dysphonia and the aging voice. Geriatric Care Otolaryngology. Alexandria: AAO-HNSF; 2006. p 67-81.

7. Belafsky PC, Postma GN. Vocal fold augmentation with calcium hydroxylapatite. Otolaryngol Head Neck Surg. 2004 Oct; 131(4):351-4. 
8. Awd ARS, Dkhil MA, Farhoud E. Fibroblasts in the human vocal fold mucosa: an ultrastructural study of different age groups. Singapore Med J. 2009 Feb; 50(2):201-7.

9. Louzeiro TRS, Lima Pontes PA, Camponês do Brasil OO. Quantitative analysis of myelinic fibers in human laryngeal nerves according to age. Rev Bras Otorrinolaringol. 2008; 74(1):45-52.

10. Kutta $H$, Knipping $S$, Claassen $H$, Paulsen $F$. Functional anatomy of the larynx from clinical points of view: part II: laryngeal mucous membrane, blood supply, innervation, lymphatic drainage, age-related changes. HNO. 2007 Aug; 55(8):661-75.

11. Ximenes Filho JA, Melo ECM, Carneiro CG, Tsuji DH, Sennes LU. Correlação entre a altura e as dimensões das pregas vocais. Rev Bras Otorrinolaringol. 2003; 3(69):371-4.

12. Ximenes Filho JA, Tsuji DH, Nascimento $\mathrm{PH}$, Sennes LU. Histologic changes in human vocal folds correlated with aging: a histomorphometric study. Ann Otol Rhinol Laryngol. 2003 Oct; 112(10):894-8. 13. Mifune E, Justino VSS, Camargo Z, Gregio F. Análise acústica da voz do idoso: caracterização da freqüência fundamental. Rev CEFAC. 2007 Abr-Jun; 9(2):238-47.

14. Wan $P$, Huang $Z$. Effect of age and gender to voice quality. Lin Chung $\mathrm{Er} \mathrm{Bi} \mathrm{Yan} \mathrm{Hou} \mathrm{Tou} \mathrm{Jing}$ Wai Ke Za Zhi. 2007 Oct; 21(20):929-31.

15. Kosztyła-Hojna B. The evaluation of voice and aerodynamic activity of larynx in patients with vocal cords atrophy. Pol Merkur Lekarski. 2005 Feb; 18(104):151-5.

16. Shingo T, Miwako K, Takaharu N, Hiroshi I, Ken-Ichi S, Niro T. Clinical analysis of presbylarynxVocal fold atrophy in elderly individuals. Auris Nasus Larynx [Article in Press, Corrected Proof].2009 Dec [cited 2010 Mar 5]. Available form: http:// www.sciencedirect.com/science/article/B6T4M4Y1MS6N-3/2/2c292c46eb8b4b35a5e2bc13e75 $7 \mathrm{f} 4 \mathrm{f3}$.

17. Cerceau JSB, Alves CFT, Gama ACC. Análise acústica da voz de mulheres idosas. Rev CEFAC. 2009 Jan-Mar;11(1):142-9.

18. Shaheen NA. The aging female voice: Acoustic and respiratory data Clinical Linguistics \& Phonetics. 2006; 20(2-3):171-80.

19. Thomas LB, Harrison AL, Stemple JC. Aging thyroarytenoid and limb skeletal muscle: lessons in contrast. J Voice. 2008 Jul; 22(4):430-50.

20. Cassol M. Avaliação da Percepção do Envelhecimento Vocal em Idosos. Estud. interdiscip. envelhec. 2006; 9:41-52.

21. Menezes LN, Vicente LCC. Envelhecimento vocal em idosos institucionalizados. Rev. CEFAC. 2007 Jan-Mar; 9 (1):90-8.

22. Cohen SM, Turley R. Coprevalence and impact of dysphonia and hearing loss in the elderly. Laryngoscope. 2009 Sep;119(9):1870-3.

23. Kendall K. Presbyphonia: a review. Speech therapy and rehabilitation. Otolaryngol Head Neck Surg. 2007 Jun; 15(3):137-40.

doi: 10.1590/S1516-18462011005000029

RECEBIDO EM: 19/01/2010

ACEITO EM: 24/03/2010

Endereço para correspondência:

Sandrelli Virginio de Vasconcelos Miranda

Rua 01, 115

Vitória de Santo Antão - PE

CEP: 55608-430

E-mails: sandrelli@gmail.com sandrelliv@ hotmail.com 